\title{
A Rare Complication after Latissimus Dorsi Flap Breast Reconstruction: Radial Nerve Palsy
}

\author{
Joon Hyun Kwon, Jae Bong Kim, \\ Jeong Woo Lee, Kang Young Choi, \\ Ho Yun Chung, Byung Chae Cho, \\ Jung Dug Yang
}

Department of Plastic and Reconstructive Surgery, Kyungpook National University School of Medicine, Daegu, Korea

No potential conflict of interest relevant to this article was reported.

\begin{abstract}
Among autologous breast reconstruction techniques, breast reconstruction using the latissimus dorsi musculocutaneous flap is widely used, offering advantages including the relative simplicity of the procedure and the reliable and consistent vascularity of the flap. Accordingly, more than 500 cases have been performed in the past 8 years at Kyungpook National University Medical Center. This study reports on a rare case involving a radial nerve neuropathy complication which was experienced for the first time at the medical center. The current case demonstrates that in addition to common complications, such as seroma of the donor site and scarring, additional intraoperative complications in areas unrelated to the surgical site can occur, including radial nerve neuropathy in the opposite arm.
\end{abstract}

Keywords Mammaplasty, Radial neuropathy, Superficial back muscles

\section{INTRODUCTION}

In the past 10 years, continuous advancements have been made in breast reconstruction techniques, in addition to therapeutic mastectomy for breast cancer, while paying careful attention to aesthetics. Among various breast reconstruction techniques, breast reconstruction with the latissimus dorsi (LD) musculocutaneous flap, first introduced in the mid-1970s by Schneider et al. [1] and Olivari [2], has been widely used because of advantages associated with being a relatively simple surgical technique, while overcoming insufficient volume and skin [3].

Needless to say, there are potential postoperative complications, but compared to other surgical techniques, it has a very low risk and incidence rate of complications. Because breast reconstruction with the LD musculocutaneous flap has a relatively smaller surgical range than others, postoperative scars are more favorable that

Received: Aug 27, 2016 Revised: Oct 21, 2016 Accepted: Oct 24, 2016 Correspondence: Jung Dug Yang

Department of Plastic and Reconstructive Surgery, Kyungpook National University School of Medicine, 130, Dongdeok-ro, Jung-gu, Daegu 41944, Korea. E-mail: lambyang@daum.net

Copyright @ 2016 The Korean Society for Aesthetic Plastic Surgery.

This is an Open Access article distributed under the terms of the Creative Commons Attribution Non-Commercial License (http://creativecommons.org/licenses/by-nc/4.0/) which permits unrestricted non-commercial use, distribution, and reproduction in any medium, provided the original work is properly cited. www.e-aaps.org those from other surgical methods, and it also offers a lower risk of complications such as infection and hematoma owing to its relatively shorter operating duration [4].

Complications like seroma, scarring, infection, and breast animation have been reported by other surgeons as a common occurrence. Herein, information is shared on a case of radial nerve neuropathy which occurred for the first time among more than 500 cases of LD flap breast reconstruction performed over the past 8 years at Kyungpook University Medical Center.

\section{CASE}

A 51-year-old female patient visited Kyungpook University Medical Center after being diagnosed with ductal carcinoma in situ (DCIS) in the left (Lt.) breast from a biopsy performed at another hospital. The department of surgery scheduled a skin sparing mastectomy (SSM) and the patient was referred to the department of plastic surgery for consultation on breast reconstruction. After consulting with a plastic surgeon, volumetry was performed and the decision was made to use a LD flap breast reconstruction technique suitable for moderate-sized breasts (right [Rt.] $180 \mathrm{cc}$, Lt. $185 \mathrm{cc}$ ) which met the patient's needs. Afterwards, preoperative photos, chest computed tomography (CT) angiography, and the shoulder's range of motion (ROM) were assessed according to protocol. The surgery was performed by the surgical department and involved a SSM in the supine position, followed by LD flap elevation and tunneling after 
changing to the Rt. decubitus position in order to move into the site of skin and soft tissue defects. The patient was returned to the supine position to perform the breast reconstruction while the position of the LD flap was adjusted. There were no specific findings

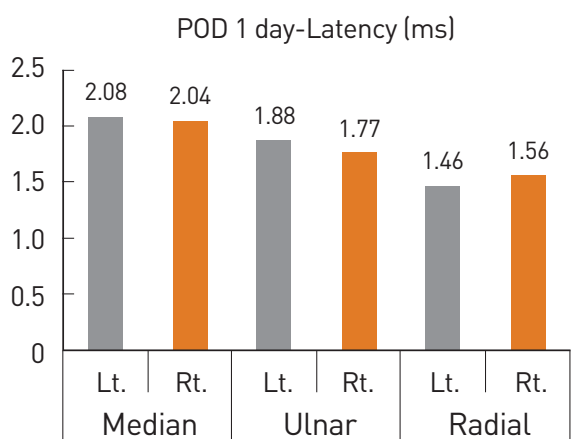

POD 6 months-Latency (ms)

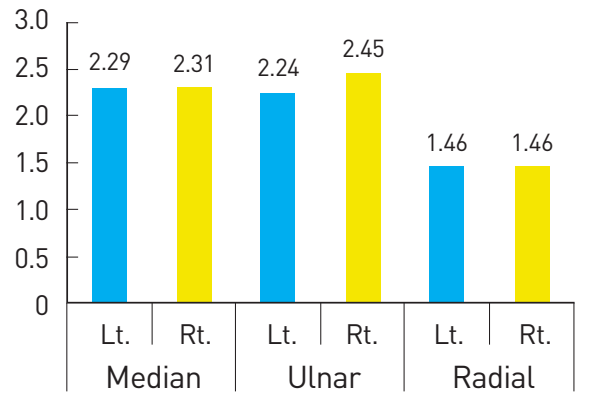

POD 1 day-Lat. Pk (ms)

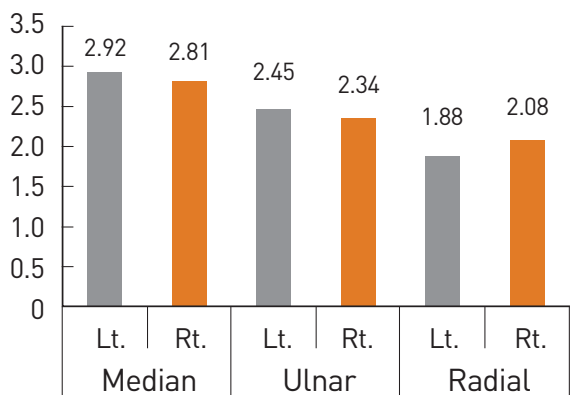

POD 6 months-Lat. Pk (ms)

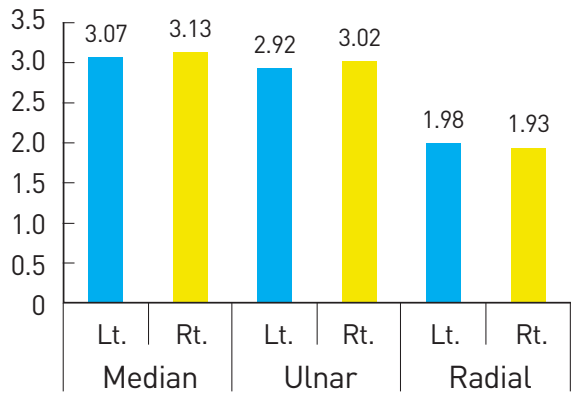

POD 1 day-Amp. $(\mu \mathrm{V})$

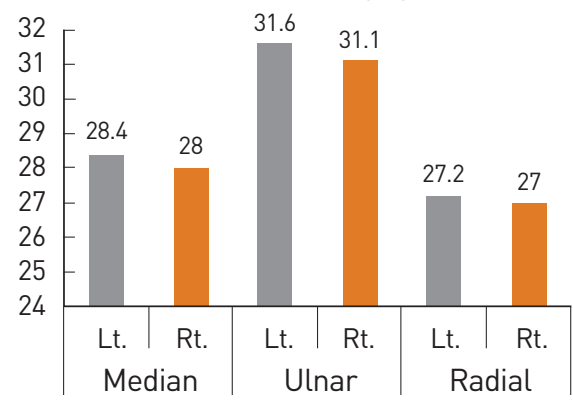

POD 6 months-Amp. $(\mu \mathrm{V})$

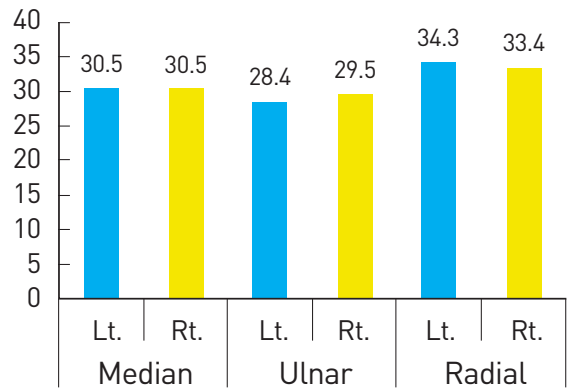

Fig. 1. Sensory nerve conduction study on postoperative day 1 and at 6 months. POD, postoperative day; Rt., right; Lt., left; Amp, amplitude; Lat. $\mathrm{Pk}$, latency peak.

POD 1 day-Latency (ms)

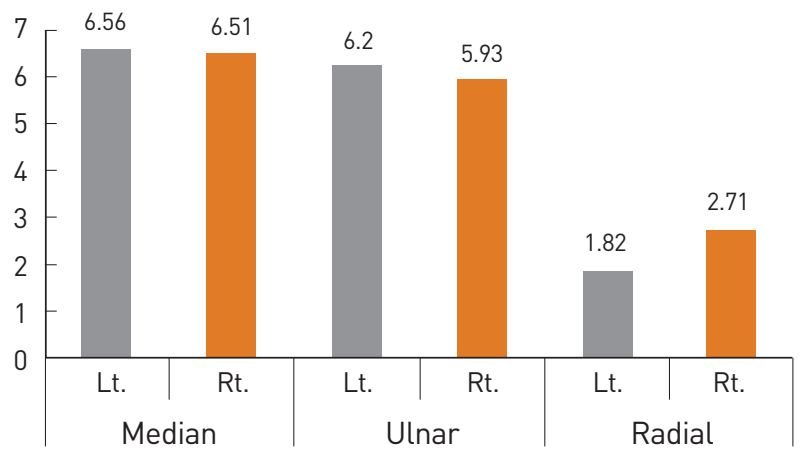

POD 6 months-Latency (ms)

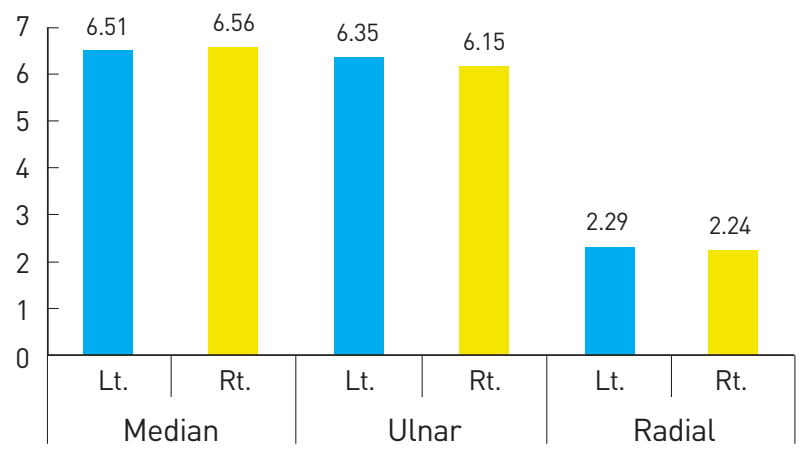

POD 1 day-Amp (mV)

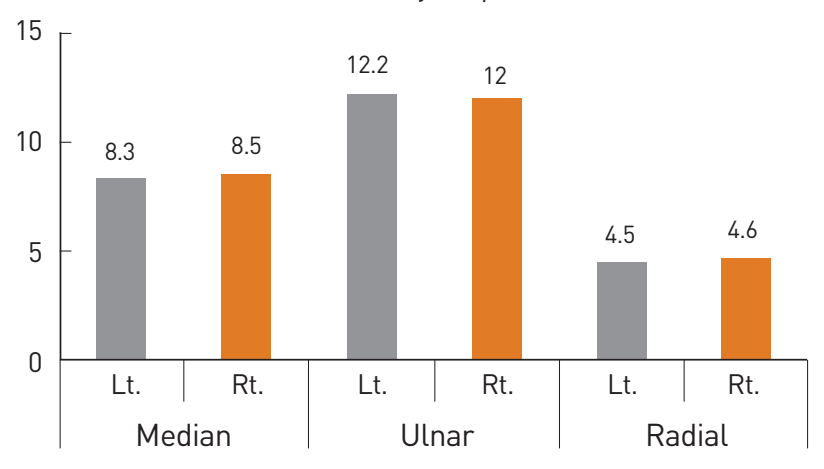

POD 6 months-Amp (mV)

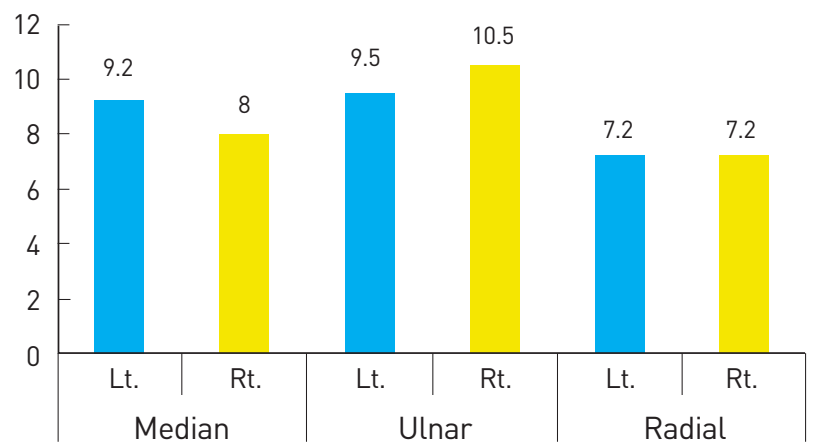

Fig. 2. Motor nerve conduction study on postoperative day 1 and at 6 months. POD, postoperative day; Rt., right; Lt., left; Amp, amplitude. 
in the surgical sites on the Lt. breast and donor site following the surgery; however, abnormal findings, including numbness, wrist drop and a tingling sensation in the Rt. arm were found starting at postoperative day (POD) 1. Electromyography (EMG) was performed to check for abnormalities, and a difference of $\geq 20 \%$ between the EMG measurements from the Lt. And Rt. sides suggested nerve abnormalities.

In Fig. 1, all cases were determined to be normal since there were no cases showing a difference of $\geq 20 \%$ in latency, latency peak, and amplitude between the Lt. and Rt. sides on the sensory nerve conduction study (NCS) performed on POD 1. However, there were abnormal findings, as shown in Fig. 2, where there was a difference of $\geq 44 \%\left(2.71 / 1.88^{\star} 100\right)$ in latency values between the Lt. and Rt. sides on the motor NCSs performed on POD1.

Moreover, abnormal findings in the positive sharp wave (PSW) of the needle EMG appeared in the external carpi radialis longus, external indicis, and brachioradialis of the Rt. arm (Table 1). Considering the muscles showing abnormal findings, a diagnosis was made of radial nerve neuropathy caused by injury to the radial nerve level.

Based on the surface anatomy, it was suspected that compressive radial nerve neuropathy had occurred due to unstable positioning during the LD flap breast reconstruction, which required several position changes during the procedure (Fig. 3).

Afterwards, aggressive pain control with medication (maxnophen $32 \mathrm{mg} / 37.5 \mathrm{mg}$ qd, gabapentin $300 \mathrm{mg}$ tid), complex exercise,

Table 1. Needle electromyography summary table for postoperative day 1 and at 6 months

\begin{tabular}{|c|c|c|c|c|c|c|c|c|c|c|c|c|c|c|c|c|c|c|}
\hline & \multicolumn{9}{|c|}{ POD 1} & \multicolumn{9}{|c|}{6 months } \\
\hline & IA & Fib & PSW & Fasc & H.F. & Amp & Dur. & PPP & $\frac{\text { Recruit. }}{\text { Pattern }}$ & IA & Fib & PSW & Fasc & H.F. & Amp & Dur. & PPP & $\frac{\text { Recruit. }}{\text { Pattern }}$ \\
\hline Rt. first dorsal inteross & $\mathrm{N}$ & $\mathrm{N}$ & $\mathrm{N}$ & $\mathrm{N}$ & N & $\mathrm{N}$ & $\mathrm{N}$ & $\mathrm{N}$ & $\mathrm{N}$ & $\mathrm{N}$ & $\mathrm{N}$ & $\mathrm{N}$ & $\mathrm{N}$ & $\mathrm{N}$ & N & $\mathrm{N}$ & $\mathrm{N}$ & N \\
\hline Rt. abductor poll brevis & $\mathrm{N}$ & $\mathrm{N}$ & $\mathrm{N}$ & $\mathrm{N}$ & N & $\mathrm{N}$ & $\mathrm{N}$ & $\mathrm{N}$ & $\mathrm{N}$ & $\mathrm{N}$ & $\mathrm{N}$ & $\mathrm{N}$ & $\mathrm{N}$ & $\mathrm{N}$ & N & $\mathrm{N}$ & $\mathrm{N}$ & N \\
\hline Rt. flex carpi RAD & $\mathrm{N}$ & $\mathrm{N}$ & $\mathrm{N}$ & $\mathrm{N}$ & N & $\mathrm{N}$ & $\mathrm{N}$ & $\mathrm{N}$ & $\mathrm{N}$ & $\mathrm{N}$ & $\mathrm{N}$ & N & N & $\mathrm{N}$ & N & $\mathrm{N}$ & $\mathrm{N}$ & N \\
\hline Rt. external indicis & $\mathrm{N}$ & +1 & +1 & $\mathrm{~N}$ & N & $\mathrm{N}$ & $\mathrm{N}$ & p.p. & D & $\mathrm{N}$ & $\mathrm{N}$ & $\mathrm{N}$ & N & $N$ & N & $\mathrm{N}$ & p.p. & D \\
\hline Rt. brachioradialis & $\mathrm{N}$ & $\mathrm{N}$ & +1 & $\mathrm{~N}$ & N & $\mathrm{N}$ & $\mathrm{N}$ & p.p. & D & $\mathrm{N}$ & $\mathrm{N}$ & $\mathrm{N}$ & $\mathrm{N}$ & $N$ & N & $\mathrm{N}$ & p.p. & $\mathrm{D}$ \\
\hline Rt. biceps & $\mathrm{N}$ & $N$ & $\mathrm{~N}$ & $\mathrm{~N}$ & $N$ & $\mathrm{~N}$ & $\mathrm{~N}$ & N & $\mathrm{N}$ & $\mathrm{N}$ & $\mathrm{N}$ & $\mathrm{N}$ & $\mathrm{N}$ & $N$ & N & $\mathrm{N}$ & $\mathrm{N}$ & $N$ \\
\hline Rt. triceps & $\mathrm{N}$ & $\mathrm{N}$ & $\mathrm{N}$ & $\mathrm{N}$ & $\mathrm{N}$ & $\mathrm{N}$ & $\mathrm{N}$ & $\mathrm{N}$ & $\mathrm{N}$ & $\mathrm{N}$ & $\mathrm{N}$ & $\mathrm{N}$ & $\mathrm{N}$ & $\mathrm{N}$ & N & $\mathrm{N}$ & $\mathrm{N}$ & N \\
\hline Rt. deltoid & $\mathrm{N}$ & $\mathrm{N}$ & $\mathrm{N}$ & $\mathrm{N}$ & N & $\mathrm{N}$ & $\mathrm{N}$ & $\mathrm{N}$ & $\mathrm{N}$ & $\mathrm{N}$ & $\mathrm{N}$ & $\mathrm{N}$ & $\mathrm{N}$ & $N$ & N & $\mathrm{N}$ & $\mathrm{N}$ & N \\
\hline
\end{tabular}

POD, postoperative day; MUAP, motor unit action potential; IA, insertion activity; Fib, fibrillation; PSW, polyspike wave; Fasc, fasciculation; H.F., high frequency; Amp, amplitude; Dur., durations; PPP, polyphasic potential; Rt., right; RAD, radialis; N, normal; D, decreased.
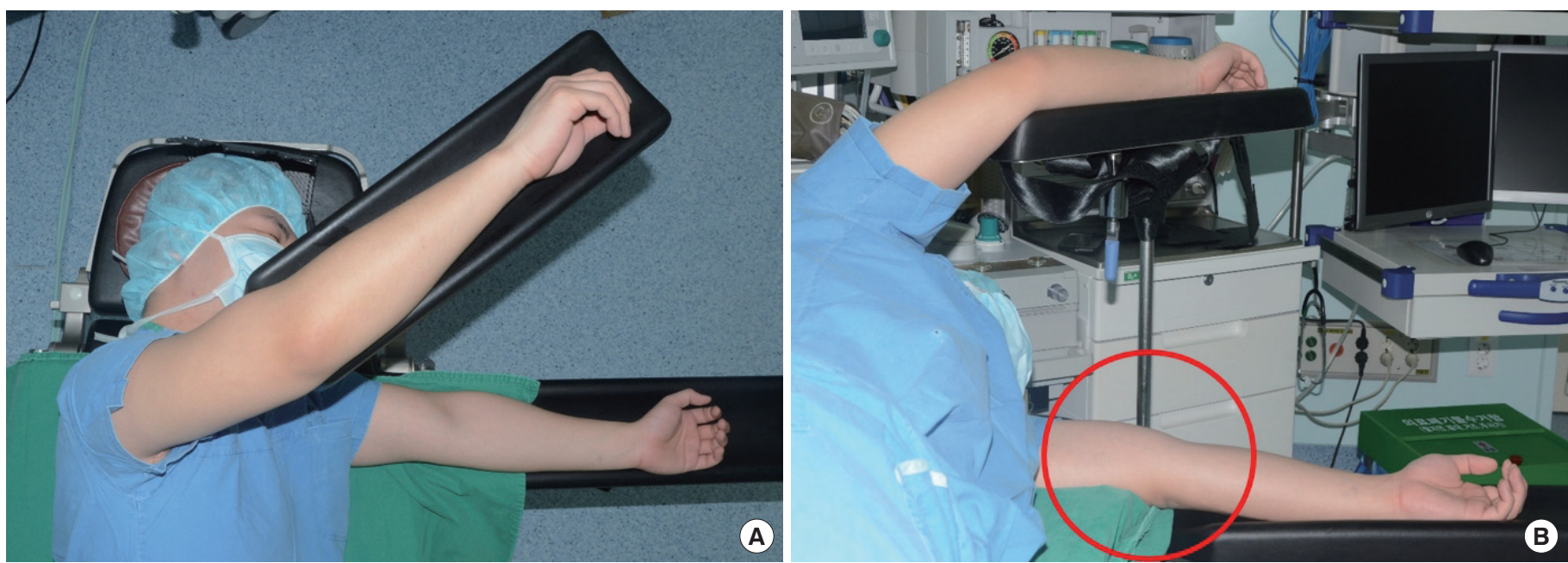

Fig. 3. Posture and unstable position in the decubitus position during the latissimus dorsi flap breast reconstruction. (A) Above view. (B) The decubitus position can lead to radial groove's compression. 
and splint application were implemented with consultation from the department of rehabilitative medicine. As a result, from POD 1 month on, sensory problems including numbness and a tingling sensation were resolved; from POD 3 months on, motor problems including wrist drop also showed improvement; and by POD 6 months, complete recovery was achieved. EMG performed at POD 6 months showed normal findings (Table 1, Fig. 1 and 2), and since then, the patient has shown no further abnormal findings.

\section{DISCUSSION}

LD flap breast reconstruction is a procedure that uses autologous tissues and is often used for breast reconstruction following a mastectomy among Asian women with small to moderate-sized breasts $[4,5]$. The surgical technique has advanced over time with many surgeons choosing to use the procedure, and reports on complications associated with the procedure have been decreasing [6-8]. However, the present case demonstrates that perioperative complications, such as radial nerve neuropathy, can also occur in the opposite arm, not only at the surgical site.

The radial nerve originates from the brachial plexus and supplies the posterior portion of the upper limbs, and is involved in innervation of the 12 muscles responsible for extension of the wrists and hands (Fig. 4A) [9]. Moreover, the superficial radial nerve branch is responsible for sensory functions of the hand dorsum site. The radial nerve is one of the major nerves that is injured most frequently in the upper extremities. Radial nerve neuropathy, sometimes referred to as "honeymoon palsy," occurs from compression of the arm which injures the radial nerve, causing problems with the supinator and extension of the elbow and wrist. The symptoms may appear differently depending on the level of injury to the radial nerve (Fig. 4B).

Nerve injury caused mainly by compression can be classified into demyelinating, axonal, and neurotmesis types (Table 2). Among these, the demyelinating and axonal types, which do not cause nerve continuity problems, have different periods required for recovery depending on the extent of the nerve injury. Moreover, most cases show complete recovery with just conservative treatments such as pain control, complex exercise, and splint application. However, for the neurotmesis type, which causes nerve continuity problems due to trauma (e.g., humerus fractures), recovery with just conservative treatments cannot be expected; thus, aggressive treatments such as surgical interventions are needed, and sequelae can remain after treatment [10].

The present case demonstrated a nerve injury on the opposite arm, unrelated to the surgical site. Thus, peripheral neuropathy such as radial nerve neuropathy can occur at any time if persistent compression is applied to the upper arm by surgical or other devices during the several position changes required in LD flap breast reconstruction. As shown in Fig. 5, gel padding can be used as prevention on the area where compression may occur, and this measure should be able to help prevent such complications from occurring [11].

Table 2. Nerve injury classification and treatment strategy

\begin{tabular}{lccc}
\hline & Demyelinating & Axonal & Neurotmesis \\
\hline Injury & Noloss & Axon & Neural tube \\
Recovery time & 2-4 weeks & Several months & Permanent \\
Treatment & Conservative & Conservative & Surgical intervention \\
\hline
\end{tabular}

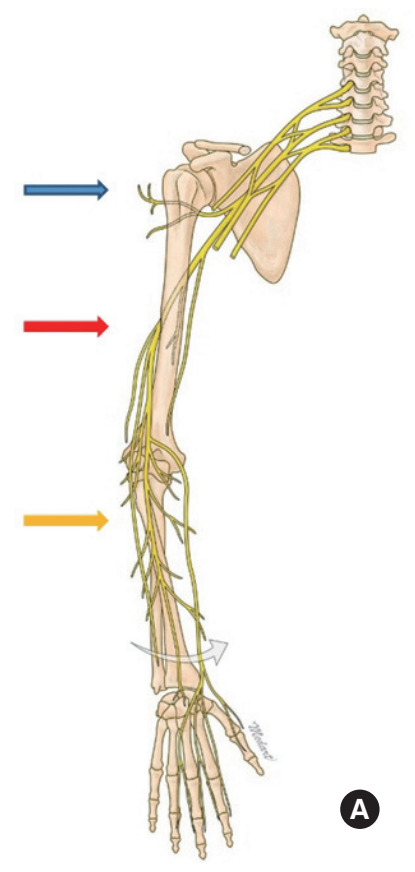

\begin{tabular}{|c|c|c|c|c|}
\hline & Forearm level & Radial level & Groove & Axilla level \\
\hline \multicolumn{5}{|l|}{ Motor } \\
\hline Wrist drop or finger drop & + & + & & + \\
\hline Diminished brachioradialis & - & + & & + \\
\hline Weakness of elbow extension & - & - & & + \\
\hline \multicolumn{5}{|l|}{ Sensory } \\
\hline Loss in lateral dorsal hand & - & + & & + \\
\hline Loss in posterior arm & - & - & & + \\
\hline
\end{tabular}

Fig. 4. (A) Level of radial nerve injury. (B) Symptoms based on injury levels. blue arrow, axilla level; red arrow, radial groove level; yellow arrow, forearm level. 


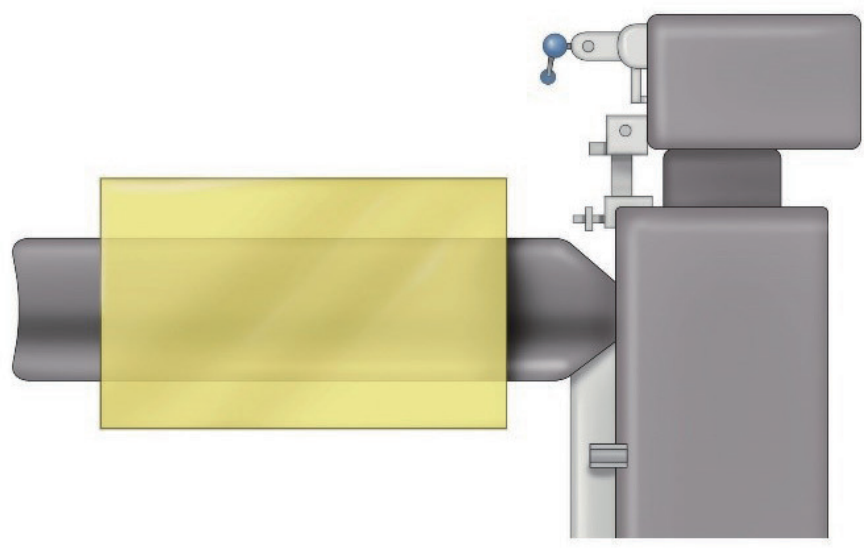

Fig. 5. Positioning of gel padding during surgery.

Looking at the present case from a broader perspective, intraoperative complications can occur not only at surgery-related areas, but also in unexpected areas, such as radial nerve neuropathy in the opposite arm; therefore, close attention should be paid to the smallest of patient-related issues during all surgical procedures.

\section{PATIENT CONSENT}

Patients provided written consent for the use of their images.

\section{REFERENCES}

1. Schneider WJ, Hill HL Jr, Brown RG. Latissimus dorsi myocutaneous flap for breast reconstruction. Br J Plast Surg 1977;30:277-81.

2. Olivari N. The latissimus flap. Br J Plast Surg 1976;29:126-8.

3. Hammond DC. Latissimus dorsi flap breast reconstruction. Clin Plast Surg 2007;34:75-82.

4. Chang DW, Youssef A, Cha S, et al. Autologous breast reconstruction with the extended latissimus dorsi flap. Plast Reconstr Surg 2002;110:

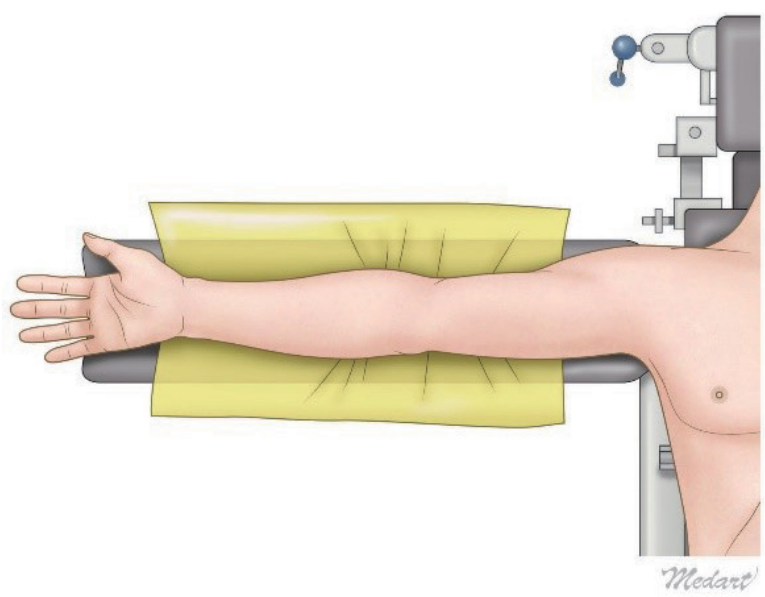

751-9; discussion 760-1.

5. Yang JD, Kim MC, Lee JW, et al. Usefulness of oncoplastic volume replacement techniques after breast conserving surgery in small to moderate-sized breasts. Arch Plast Surg 2012;39:489-96.

6. Hammond DC. Latissimus dorsi flap breast reconstruction. Plast Reconstr Surg 2009;124:1055-63.

7. Roy MK, Shrotia S, Holcombe C, et al. Complications of latissimus dorsi myocutaneous flap breast reconstruction. Eur J Surg Oncol 1998; 24:162-5.

8. Germann G, Steinau HU. Breast reconstruction with the extended latissimus dorsi flap. Plast Reconstr Surg 1996;97:519-26.

9. Robson AJ, See MS, Ellis H. Applied anatomy of the superficial branch of the radial nerve. Clin Anat 2008;21:38-45.

10. Lubahn JD, Cermak MB. Uncommon nerve compression syndromes of the upper extremity. J Am Acad Orthop Surg 1998;6:378-86.

11. Blackburn A, Taghizadeh R, Hughes D, et al. Prevention of perioperative limb neuropathies in abdominal free flap breast reconstruction. J Plast Reconstr Aesthet Surg 2016;69:48-54. 